\title{
Evaluación de la energía mareomotriz en el norte del Golfo de California con fines de generación de energía eléctrica
}

\section{Tidal energy evaluation in the north of the Gulf of California for power generation purposes}

QUIÑONEZ-OSUNA, José Ramón†*, MARINONE-MOSCHETTO, Silvio Guido, FLORESAGUILAR, María Dolores y CARMONA-DUARTE, Domingo

Instituto Tecnológico de Mazatlán

ID 1 $1^{\mathrm{er}}$ Autor: José Ramón, Quiñonez-Osuna / ORC ID: 0000-0001-9182-6171

ID $1^{\mathrm{er}}$ Coautor: Silvio Guido, Marinone-Moschetto / ORC ID: 0000-0002-0294-9186

ID $2^{\text {do }}$ Coautor: María Dolores, Flores-Aguilar / ORC ID: 0000-0002-2017-9197

ID $3^{\text {er }}$ Coautor: Domingo, Carmona-Duarte

DOI: $10.35429 / J E E .2019 .8 .3 .33 .39$

Recibido Febrero 20, 2019; Aceptado Marzo 30, 2019

\section{Resumen}

Las corrientes de marea constituyen una fuente limpia e inagotable de energía. Determinar su magnitud en la zona norte del Golfo de California con miras a su aprovechamiento, representó el objeto central de este trabajo. La metodología comprendió una búsqueda exhaustiva de mediciones de corrientes de marea, la generación del archivo de texto de parejas ordenadas tiempo-velocidad (historial de velocidades a manera de tabulación) a partir de los gráficos de velocidades encontrados, y finalmente su procesamiento, lo cual consistió en la obtención de los historiales de densidad de potencia de la corriente y de potencia generada por una turbina estándar, así como las gráficas correspondientes de frecuencia y equivalencia respecto a una planta de generación continua. Los resultados obtenidos muestran que la energía disponible en las corrientes de marea de la región norte del Golfo de California resulta inferior que la de otras zonas del mundo con cambios radicales de marea, sin embargo se considera que la instalación de varias granjas en lugar de una sola, situadas en los sitios donde las corrientes alcanzan mayores velocidades, aunado al aprovechamiento de otras fuentes renovables disponibles en la región, constituyen en conjunto una opción importante que se debe considerar.

Energía mareomotriz, Central mareomotriz, Sitios con cambios radicales de marea

\begin{abstract}
The tidal currents constitute a clean and inexhaustible source of energy. The determination of its magnitude in the north of the Gulf of California with a view to its use, represented the central objective of this work. The methodology included an exhaustive search of measurements of tidal currents, the generation of a text file of ordered time-speed data (history of speeds as a tabulation) from the found graphs of speeds, and finally its processing, which consisted of the histories of the current power density and power generated by a standard turbine, as well as the corresponding graphs of frequency and equivalence with respect to a continuous generation plant. The results show that the energy available in the tidal currents of the northern region of the Gulf of California is lower than that of other areas of the world with radical tidal changes; however, it is considered that the installation of several farms instead of one, located in the places where the currents reach higher speeds, together with the use of other renewable sources available in the region, constitute altogether an important option that must be considered.
\end{abstract}

Tidal energy, Tidal power plant, Locations of largest tidal ranges

Citación: QUIÑONEZ-OSUNA, José Ramón, MARINONE-MOSCHETTO, Silvio Guido, FLORES-AGUILAR, María Dolores y CARMONA-DUARTE, Domingo. Evaluación de la energía mareomotriz en el norte del Golfo de California con fines de generación de energía eléctrica. Revista de Ingeniería Eléctrica. 2019. 3-8: 33-39.

$\dagger$ Investigador contribuyendo como primer Autor.

*Correspondencia al Autor correo electrónico: (jrquinonez@itmazatlan.edu.mx) 


\section{Introducción}

La atmósfera de la Tierra se encuentra en una condición seria de deterioro, la necesidad mundial de duplicar en 15 años la producción de energía eléctrica involucra un severo desequilibrio en caso de no llevar a cabo mejoras sustanciales en los métodos de generación, según lo establece la Agencia Internacional de Energía (IEA). Por ello, a partir de mediciones de velocidad realizadas a $20 \mathrm{~m}$. de profundidad por el CICESE (Centro de Investigación Científica y de Educación Superior de Ensenada), a lo largo de un ciclo completo de marea, se evalúa la potencia generada por una turbina mareomotriz estándar, en un sitio localizado al sureste de San Felipe Baja California (Figura 1).

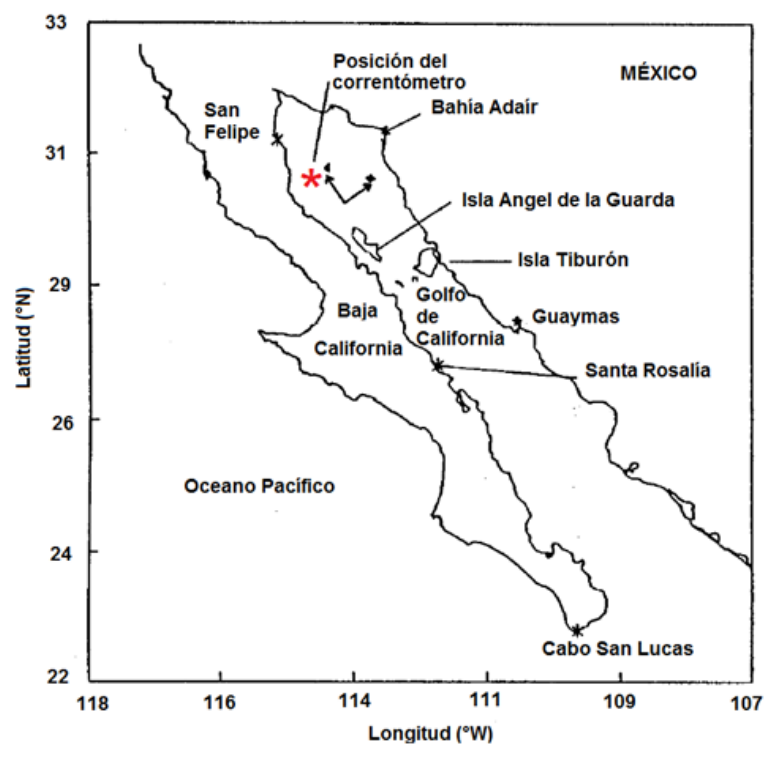

Figura 1 Localización del correntómetro empleado por los investigadores del CICESE para obtener registros longitudinales y transversales de velocidad de las corrientes de marea en el Golfo de California

Para ello fue necesario llevar a cabo una búsqueda de mediciones de velocidad de corrientes de marea, en zonas viables de la zona Golfo de California. Las mediciones fueron conseguidas a manera de 2 gráficas: una de velocidades longitudinales (a lo largo del golfo) y otra de velocidades transversales (perpendiculares a las primeras). Las gráficas fueron obtenidas por el Dpto. de Oceanografía Física del CICESE en marzo de 1988 [1]. Las mediciones fueron realizadas en lapsos de una hora, a una profundidad de $20 \mathrm{~m}$. durante un periodo de marea muy parecido al correspondiente del 2018, como puede apreciarse en el gráfico 1. La gráfica superior corresponde a marzo 1988 y la inferior a marzo 2018.

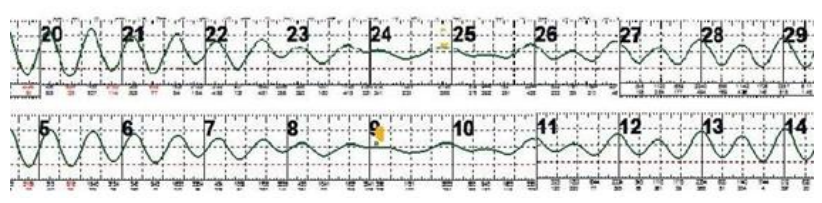

Gráfico 1 Gráficas comparativas de mareas de los meses de marzo 1988 (grafica superior) y marzo 2018 (gráfica inferior) [2]

Por otra parte, después de una revisión bibliográfica sobre los métodos existentes para extraer la energía de las mareas y de las tecnologías empleadas por cada uno, fue seleccionado el método de corrientes de mareas, empleando turbinas situadas perpendiculares al flujo, por su bajo daño al medio ambiente, menor costo y buena eficiencia.

Las gráficas de velocidad aportadas por el CICESE se muestran en el Gráfico 2. Las características del sitio de medición son las siguientes: diferencia máxima de mareas: 6m; tipo de marea: semidiurna (dos mareas altas diferentes y dos mareas bajas diferentes); profundidad: $100 \mathrm{~m}$; anchura del golfo: $5 \mathrm{~km}$.

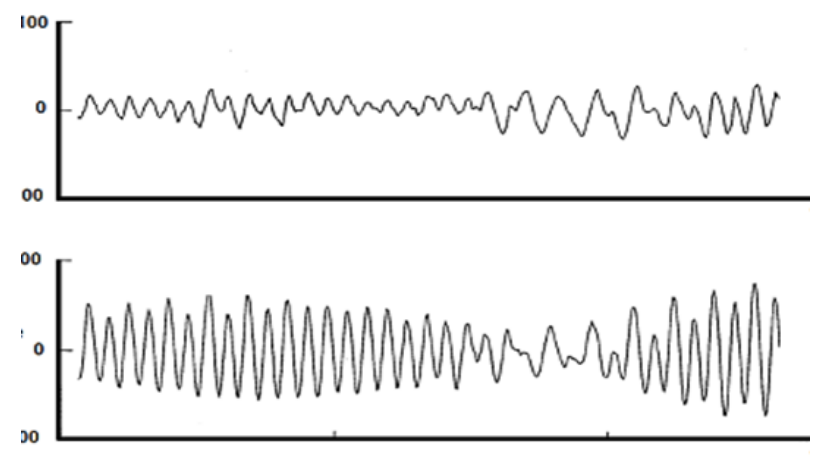

Gráfico 2 Valores de velocidad transversal (gráfica superior) y longitudinal (gráfica inferior) en $\mathrm{cm} / \mathrm{seg}$, aportados por el CICESE, correspondientes al mes de marzo 1988

El EPRI (Electric Power Research Institute) establece que mediciones de corriente como las mostradas en la Figura 3 son confiables por un periodo de 30 años [3]. Así mismo, se señala que existe muy diversa información sobre el aprovechamiento de la energía de las mareas en México, y particularmente en la región Norte del golfo de California, donde se presentan diferencias críticas del nivel del mar. Sin embargo, no se encontraron publicaciones basadas en mediciones continuas de velocidad. Mejía et al. [4] e Hiriart Le Bert [5] presentan para esta zona predicciones de velocidades de corrientes de marea y potencia probable de generación, basadas en modelos enteramente teóricos.

QUIÑONEZ-OSUNA, José Ramón, MARINONE-MOSCHETTO, Silvio Guido, FLORES-AGUILAR, María Dolores y CARMONADUARTE, Domingo. Evaluación de la energía mareomotriz en el norte del Golfo de California con fines de generación de energía eléctrica. Revista de Ingeniería Eléctrica. 2019. 
La energía cinética de una corriente de fluido se determina por la conocida expresión:

$$
E c=\frac{1}{2} m v^{2}
$$

Donde $\quad m$ y $\quad v \quad$ representan respectivamente la masa y la velocidad del agua. Luego, la potencia disponible en la corriente de marea que atraviesa una sección $A$ perpendicular al flujo es obtenida dividiendo la energía cinética entre el tiempo:

$$
\begin{aligned}
& P=\int_{A} \frac{E c}{t}=\int_{A} \frac{0.5 m v^{2}}{t}=\int_{A} \frac{0.5 \rho V v^{2}}{t}= \\
& \int_{A} \frac{0.5 \rho A l v^{2}}{t}=\int_{A} 0.5 \rho A v^{3}
\end{aligned}
$$

donde $P$ es la potencia, la densidad del agua de mar está dada por $\rho \approx 1.027 \mathrm{~kg} / \mathrm{m}^{3}, v$ representa la velocidad instantánea del flujo, $m$ la masa, $t$ el tiempo y $V$ el volumen. Luego, si se considera que el flujo es uniforme sobre la sección transversal $A$ entonces la expresión 2 se puede escribir como se muestra en la ecuación 3 para determinar la densidad de potencia $(P / A)$.

$$
P / A=0.5 \rho v^{3}
$$

El comportamiento de la eficiencia de una turbina estándar de corrientes se muestra en el Gráfico 3, el cual aporta la eficiencia correspondiente a cada velocidad registrada. De esta manera, la potencia generada por metro cuadrado de sección transversal del rotor de una turbina estándar, se consigue multiplicando la ecuación 3 por la eficiencia correspondiente, resultando así la ecuación 4. Es importante notar que para una turbina estándar, las velocidades por debajo de $0.5 \mathrm{~m} / \mathrm{s}$ y mayores $3.6 \mathrm{~m} / \mathrm{s}$ implican una potencia nula, como lo muestra el Gráfico 3.

$$
P / A=0.5 \eta \rho v^{3}
$$

Para el cálculo de una granja de turbinas mareomotrices con capacidad semejante a la de un generador eléctrico continuo, se consideró el área bajo la gráfica potencia-tiempo de este, a lo largo de un periodo de mareas, así como una distribución ficticia de turbinas mareomotrices, generando potencia ininterrumpidamente a lo largo del periodo, de manera que pudiera conseguirse aproximadamente la misma área.

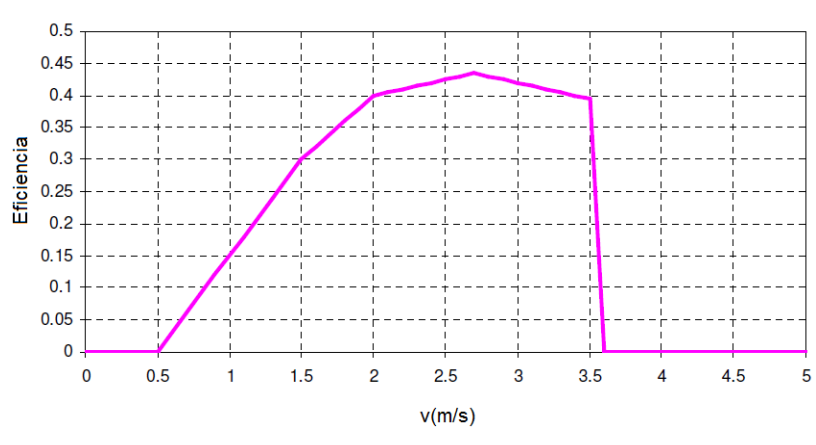

Gráfico 3 Curva de eficiencia para una turbina de corrientes de mareas típica [6]

El trabajo se compone de cuatro secciones principales: 1) Metodología, donde se presenta el método empleado para calcular la potencia a generar por una turbina estándar, 2) Historial de velocidades y elipse de corrientes de marea, que muestra la tabulación (tiempo, velocidad) elaborada a partir de las gráficas de velocidad aportadas por el CICESE (Gráfico 2), el cual representa el historial presentado a manera de parejas ordenadas, y que es indispensable de esta manera para poder evaluar tanto la energía mareomotriz como la potencia que se puede generar.

Así mismo se incluyen en esta sección, las graficas de la tabulación obtenida y su comparación con las gráficas aportadas por el CICESE, además de la elipse de corrientes de marea, la cual brinda la dirección dominante de la corriente y por tanto la posición optima de las turbinas, las cuales se colocan perpendiculares a esta dirección. La tercera sección: 3) Historiales de energía y potencia, que presenta los historiales de energía disponible en la corriente y de potencia generada por una turbina de $18 \mathrm{~m}$., los cuales permiten visualizar el grado de disponibilidad de energía eléctrica a partir de las corrientes de marea. Finamente se incluye la sección 4 que consiste en el Cálculo de una granja de turbinas mareomotrices con capacidad semejante a la de un generador eléctrico continuo

\section{Metodología}

La metodología se desarrolla de acuerdo con el siguiente procedimiento; las gráficas aportadas por el CICESE (Gráfico 2) son convertidas a un archivo de texto de tercias ordenadas tiempo velocidad longitudinal - velocidad transversal (historial de velocidades a manera de tabulación). 
Enseguida es graficada cada pareja de velocidades de la tabulación con el objeto de obtener la elipse de corrientes de marea, cuya finalidad es aportar la dirección dominante de la corriente. Posteriormente se obtiene la velocidad resultante correspondiente a cada tercia ordenada y finalmente, mediante las ecuaciones 1- 4, se determinan respectivamente, para cada tercia ordenada: energía cinética, potencia disponible en la corriente de marea, densidad de potencia y potencia generada por metro cuadrado de sección transversal de rotor, de una turbina estándar.

\section{Historial de velocidades y elipse de corrientes de marea.}

El archivo de texto de parejas ordenadas tiempovelocidad generado a partir de las gráficas de velocidad aportadas por el CICESE (Gráfico 2) se presenta en el Anexo 1. Por su parte, el Gráfico 4 muestra en color negro la gráfica del historial de velocidades, tal cual se presentó en el Gráfico 2 y en azul, la obtenida de la tabulación elaborada. La elipse de corrientes de marea (Figura 2) se obtiene al graficar la tabulación del Anexo 1.

La gráfica de velocidad resultante, su histograma de frecuencias y su gráfica de probabilidad acumulada, se muestran en los Gráficos 5, 6 y 7 respectivamente.
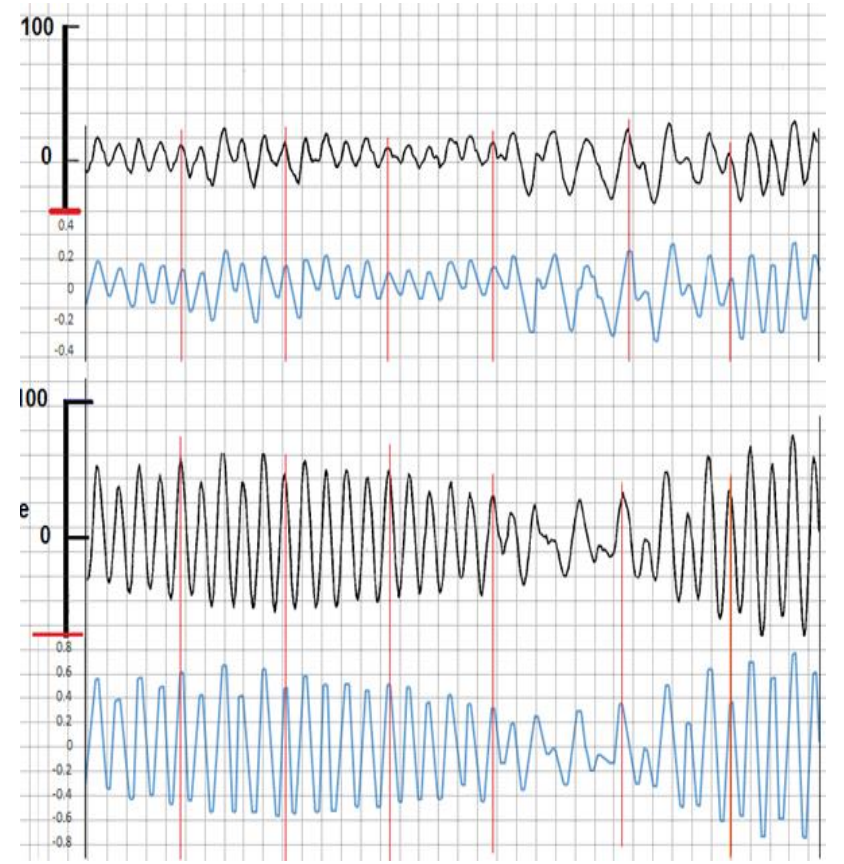

Gráfico 4 Comparación entre las graficas originales de velocidad (color negro) y las obtenidas de la tabulación generada a partir de estas (color azul)

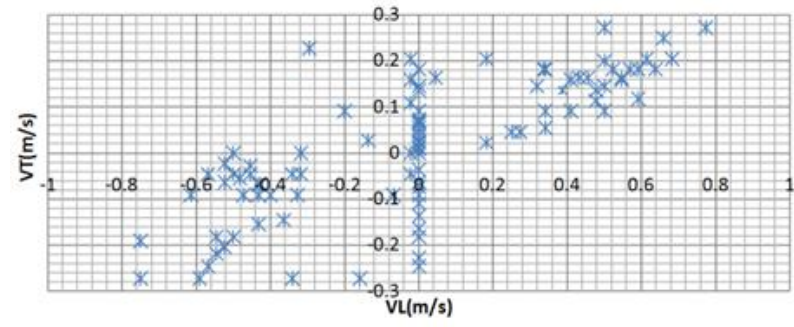

Figura 2 Elipse de la corriente de marea

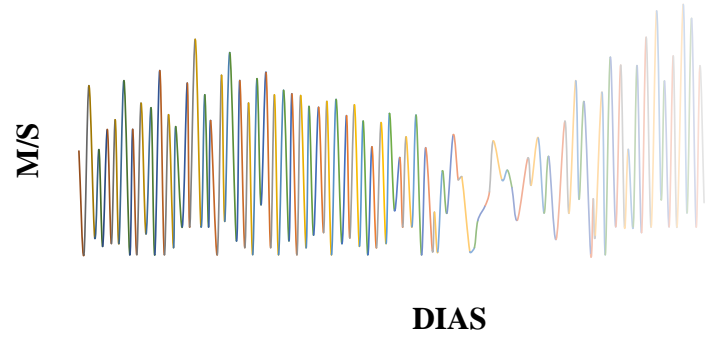

Gráfico 5 Historial de velocidad resultante

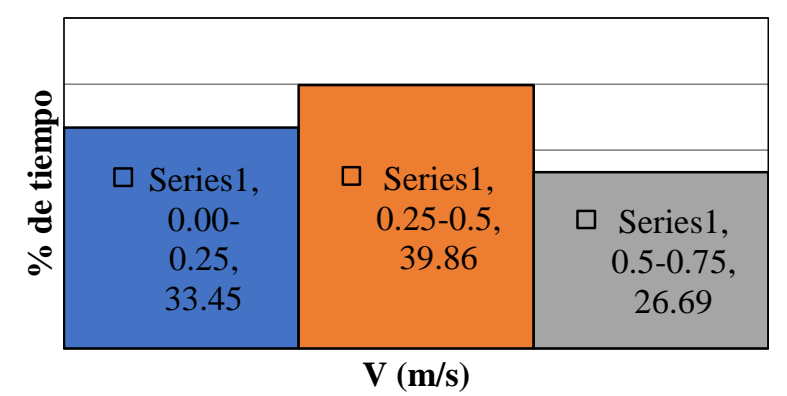

Gráfico 6 Histograma de frecuencias de velocidad

\section{Historiales de energía cinética y potencia a generar}

De acuerdo con la metodología presentada son calculados los historiales de energía cinética disponible en la corriente y de potencia generada por una turbina estándar de $18 \mathrm{~m}$. de diámetro (Gráficos 8 y 9) empleando las ecuaciones 3 y 4 respectivamente.

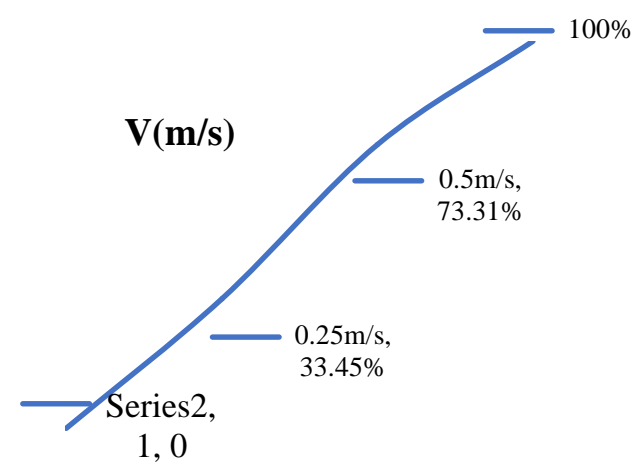

Gráfico 7 Curva de probabilidad acumulada de velocidad

QUIÑONEZ-OSUNA, José Ramón, MARINONE-MOSCHETTO Silvio Guido, FLORES-AGUILAR, María Dolores y CARMONADUARTE, Domingo. Evaluación de la energía mareomotriz en el norte del Golfo de California con fines de generación de energía eléctrica. Revista de Ingeniería Eléctrica. 2019. 


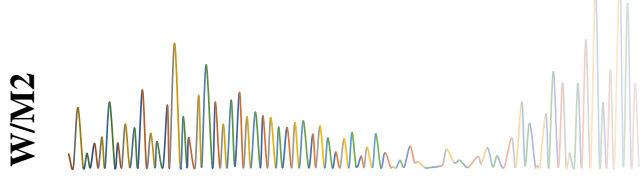

Dias

Gráfico 8 Historial de energía cinética

\section{DIAS}

Gráfico 9 Historial de potencia generada por una turbina de $18 \mathrm{~m}$. de diámetro

\section{Cálculo de una granja de turbinas mareomotrices con capacidad semejante a la de un generador eléctrico continuo}

En la Figura 3 se muestra de color rojo el área integrada bajo la curva del historial de potencia generada (gráfico 9), de manera condensada, sobreencimado en el centro de este mismo gráfico. Por su parte la Figura 4 muestra esta área y las dos parciales consideradas para conseguir una aproximación a la potencia de un generador continuo con capacidad igual a la máxima obtenida por una turbina mareomotriz de $18 \mathrm{~m}$. en el sitio analizado, es decir de $7 \mathrm{KWh}$.

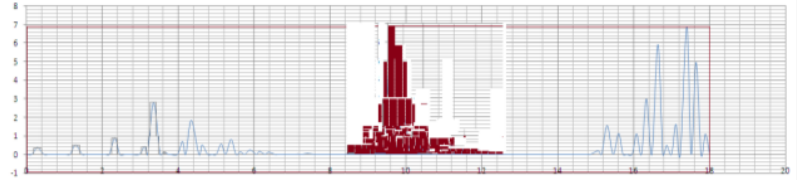

Figura 3 Área total integrada bajo la curva del historial de potencia generada por una turbina de $18 \mathrm{~m}$

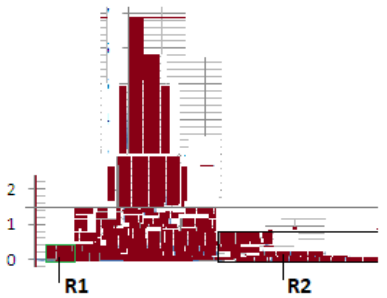

a)

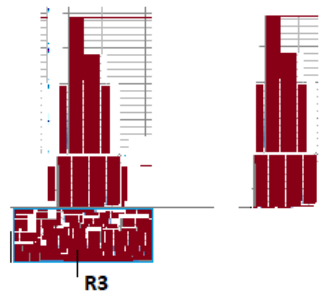

b) c)
Figura 4 Área integrada bajo la curva del historial de potencia generada por una turbina de $18 \mathrm{~m} .:$ a) área total, b) área parcial (sin regiones R1 y R2), c) área parcial (sin regiones $\mathrm{R} 1, \mathrm{R} 2$ y $\mathrm{R} 3$ )
La Figura 5 muestra el plano potenciatiempo correspondiente a un periodo de marea tal cual se mostró en el gráfico 9, cubriéndose el área correspondiente a un generador eléctrico continuo (de 18 dias por $7 \mathrm{KW}$ ), de manera aproximada en color rojo, por la producción de 20 turbinas mareomotrices (las cuales en realidad operarían de manera simultánea y no como se muestra ficticiamente, considerando 10 regiones parciales tipo b y 10 tipo c, de acuerdo con la Figura 4).

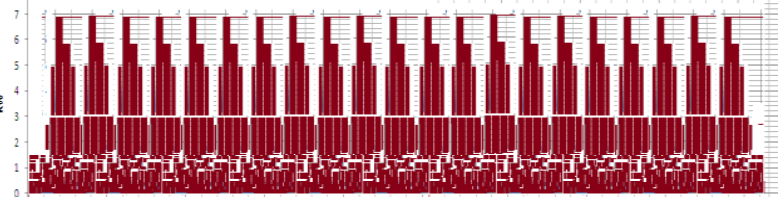

Figura 5 Producción de 20 turbinas mareomotrices, considerando 10 áreas parciales tipo b y 10 áreas parciales tipo c, de acuerdo con la Figura 13

\section{Resultados}

Con respecto de la elipse de la corriente (Figura 2), se tiene que su dirección dominante es de 5.19 grados con respecto al centro longitudinal del golfo.

En relación con el histograma de frecuencias de velocidad (Gráfico 7), así mismo se observa que en el sitio estudiado, la magnitud de la velocidad es aprovechable por aproximadamente 5 días (26.7\% del tiempo) mientras que no lo es por alrededor de 13 días.

Con referencia a la potencia generada, se puede observar en el Gráfico 9, que esta alcanza valores aceptables entre los días 2 y 6 del periodo y valores máximos los dos últimos días.

Finalmente se señala, tomando en cuenta la Figura 5, que la producción de 20 turbinas de marea de $18 \mathrm{~m}$. de diámetro equivale aproximadamente a un generador eléctrico continuo de $7 \mathrm{kwh}$.

\section{Conclusiones}

A diferencia de otros sitios en el mundo con diferencias críticas de marea, la velocidad media en el sitio analizado es reducida. Mientras que en Canadá (Quebec y Pasaje Minas) se presentan velocidades medias de la corriente de 2 a $3 \mathrm{~m} / \mathrm{s}$ [6] y en Francia (Costa Norte de Brittany) de 1.5 a $2 \mathrm{~m} / \mathrm{s}$ [7], en el sito estudiado es de $0.41 \mathrm{~m} / \mathrm{s}$.

QUIÑONEZ-OSUNA, José Ramón, MARINONE-MOSCHETTO Silvio Guido, FLORES-AGUILAR, María Dolores y CARMONADUARTE, Domingo. Evaluación de la energía mareomotriz en el norte del Golfo de California con fines de generación de energía eléctrica. Revista de Ingeniería Eléctrica. 2019. 
El presente estudio sugiere que se puede aprovechar al máximo la energía disponible en las corrientes del norte del golfo de California, donde estas muestran energía adecuada, por ejemplo en el sitio estudiado y entre las islas, donde Badan et al. [8] reportan velocidades de hasta $1.5 \mathrm{~m} / \mathrm{s}$. Es importante considerar que empleando varias granjas en vez de una sola, se puede producir en cinco días lo que no es posible el resto del periodo de marea, brindando así mismo durante estos días, una proporcional disminución de emisiones contaminantes.

En relación con la distribución de las turbinas, se recomienda sobremanera que estas debieran instalarse a distancias mayores de las consideradas en otros sitios del mundo donde las velocidades de la corriente son superiores, con el objeto de que la energía cinètica posterior a un frente de turbinas, no se vean tan afectadas.

Por otro lado se señala, que tanto la energía del viento como del sol son de magnitud adecuada en varias inmediaciones del golfo, por lo que se puede llevar a cabo un aprovechamiento en conjunto de estas tres fuentes renovables de energía.

Finalmente se desea agregar, que es de suma importancia para cualquier tipo de central de energía renovable situada a lo largo del Golfo de California, tanto el empleo de materiales resistentes al medio salino como cuidados especiales a los equipos, con el objeto de prolongar su vida útil y con ello hacer más redituable la inversión, sobre todo en aquellas granjas que solo podrían generar electricidad por periodos cortos, como es el caso de la de turbinas de marea.

\section{Agradecimientos}

Se desea agradecer al CICESE, por aportar las graficas de velocidad empleadas para desarrollar el presente trabajo

\section{Referencias}

[1] S. G. Marinone y M. F. Lavín, «Mareas y corrientes residuales en el Golfo de California,» de Contribuciones a la Oceanografía Física en México. Monografía No. 3, M. F. Lavín, Ed., Unión Geofísica Mexicana, pp. 113-139.
[2] «Calendarios,» CICESE, [En línea]. Available: predmar.cicese.mx/calendarios/. [Último acceso: 2018].

[3] G. Hagerman y B. Polagye, «EPRI North American Tidal in Stream Power Feasibility Demostration Project,» EPRI, 2006.

[4] C. J. Mejia-Olivares, I. D. Haigh, C. Neil, D. S.Coles, M. J. Lewis y S. P. Neill, «Tidal-stream energy resource characterisation for the Gulf of California, México,» Energy, 2018.

[5] G. Hiriart Le Bert, «Potencial energético de las mareas para generar electricidad,» Revista Digital Universitaria UNAM, vol. 10, nº 6, 2009.

[6] Canadian Hydraulics Center, «Guidance for Assessing Tidal Current Energy Resource,» NRC Canadian, Quebec, 2008.

[7] L. Pineau, «Tidal Streams along Brittany Northern Coast, France,» International Hydrographic Review, vol. 2, $\mathrm{n}^{\circ}$ 2, pp. 54-65, 2001.

[8] M. L. Argote, M. F. Lavín y A. Amador, «Barotropic eulerian residual circulation in the Gulf of California due to the M2 tide and wind stress,» Atmósfera, vol. 11, n 3, pp. 173-197, 1998.

\section{Anexo 1}

Archivo de texto de tiempo, velocidades longitudinales $\mathbf{y}$ tangenciales

\begin{tabular}{|r|r|r|}
\hline \multicolumn{1}{|c|}{$\mathrm{t}$ VL } & $\mathrm{VT}$ \\
\hline 0 & -0.32727273 & -0.09090909 \\
\hline 0.14376997 & 0 & 0 \\
\hline 0.28753994 & 0.52272727 & 0.18181818 \\
\hline 0.4600639 & 0 & 0.05454545 \\
\hline 0.57507987 & -0.34090909 & -0.04545455 \\
\hline 0.69009585 & 0 & 0.02727273 \\
\hline 0.81948882 & 0.38636364 & 0.13636364 \\
\hline 0.93450479 & 0 & 0.03636364 \\
\hline 1.04952077 & -0.43181818 & -0.09090909 \\
\hline 1.15015974 & 0 & -0.03636364 \\
\hline 1.29392971 & 0.54545455 & 0.16363636 \\
\hline 1.46645367 & 0 & 0 \\
\hline 1.55271565 & -0.4 & -0.09090909 \\
\hline 1.66773163 & 0 & 0 \\
\hline 1.7971246 & 0.47727273 & 0.13636364 \\
\hline 1.94089457 & 0 & 0.06818182 \\
\hline 2.08466454 & -0.47272727 & -0.09090909 \\
\hline 2.18530351 & 0 & 0 \\
\hline 2.32907348 & 0.59090909 & 0.11818182 \\
\hline 2.45846645 & 0 & 0 \\
\hline 2.58785942 & -0.43181818 & -0.15454545 \\
\hline 2.73162939 & 0 & 0.02272727 \\
\hline 2.78913738 & 0.40909091 & 0.09090909 \\
\hline 2.97603834 & 0 & -0.09090909 \\
\hline 3.11980831 & -0.52272727 & -0.20454545 \\
\hline
\end{tabular}

QUIÑONEZ-OSUNA, José Ramón, MARINONE-MOSCHETTO Silvio Guido, FLORES-AGUILAR, María Dolores y CARMONADUARTE, Domingo. Evaluación de la energía mareomotriz en el norte del Golfo de California con fines de generación de energía eléctrica. Revista de Ingeniería Eléctrica. 2019. 
Marzo 2019 Vol.3 No.8, 33-39

\begin{tabular}{|c|c|c|}
\hline 3.19169329 & 0 & -0.09090909 \\
\hline 3.34984026 & 0.65909091 & 0.25 \\
\hline 3.52236422 & 0 & 0.09090909 \\
\hline 3.62300319 & -0.52272727 & -0.02272727 \\
\hline 3.73801917 & 0 & 0.09090909 \\
\hline 3.79552716 & 0.40909091 & 0.15909091 \\
\hline 3.98242812 & 0 & 0 \\
\hline 4.11182109 & -0.54545455 & -0.21818182 \\
\hline 4.19808307 & 0 & -0.10909091 \\
\hline 4.34185304 & 0.63636364 & 0.18181818 \\
\hline 4.54313099 & 0 & 0.04545455 \\
\hline 4.62939297 & -0.56818182 & -0.04545455 \\
\hline 4.77316294 & 0 & 0.02272727 \\
\hline 4.88817891 & 0.47727273 & 0.13636364 \\
\hline 5.00319489 & 0 & 0 \\
\hline 5.13258786 & -0.54545455 & -0.18181818 \\
\hline 5.24760383 & 0 & -0.16363636 \\
\hline 5.3913738 & 0.56818182 & 0.18181818 \\
\hline 5.52076677 & 0 & 0.02272727 \\
\hline 5.63578275 & -0.52272727 & -0.02272727 \\
\hline 5.75079872 & 0 & 0 \\
\hline 5.89456869 & 0.5 & 0.2 \\
\hline 6.05271565 & 0 & 0.04545455 \\
\hline 6.13897764 & -0.52272727 & -0.06363636 \\
\hline 6.2827476 & 0 & 0 \\
\hline 6.38338658 & 0.5 & 0.14545455 \\
\hline 6.54153355 & 0 & 0.02272727 \\
\hline 6.62779553 & -0.48181818 & -0.05454545 \\
\hline 6.7571885 & 0 & 0.06363636 \\
\hline 6.90095847 & 0.45454545 & 0.16363636 \\
\hline 7.04472843 & 0 & 0.07272727 \\
\hline 7.14536741 & -0.5 & -0.04545455 \\
\hline 7.27476038 & 0 & 0 \\
\hline 7.40415335 & 0.5 & 0.09090909 \\
\hline 7.57667732 & 0 & 0.03636364 \\
\hline 7.70607029 & -0.45454545 & -0.02727273 \\
\hline 7.80670927 & 0 & 0.05454545 \\
\hline 7.93610224 & 0.47727273 & 0.11363636 \\
\hline 8.0798722 & 0 & 0.02727273 \\
\hline 8.18051118 & -0.43181818 & -0.06818182 \\
\hline 8.32428115 & 0 & 0 \\
\hline 8.43929712 & 0.34090909 & 0.09090909 \\
\hline 8.5686901 & 0 & 0.02272727 \\
\hline 8.69808307 & -0.42727273 & -0.06818182 \\
\hline 8.84185304 & 0 & 0.03636364 \\
\hline 8.94249201 & 0.43181818 & 0.16363636 \\
\hline 9.08626198 & 0 & 0.14545455 \\
\hline 9.24440895 & -0.31818182 & 0 \\
\hline 9.33067093 & 0 & 0.09090909 \\
\hline 9.41693291 & 0.34090909 & 0.18181818 \\
\hline 9.58945687 & 0 & 0.09090909 \\
\hline 9.70447284 & -0.45454545 & -0.04545455 \\
\hline 9.86261981 & 0 & 0 \\
\hline 9.99201278 & 0.31818182 & 0.14545455 \\
\hline 10.1789137 & 0 & 0.01363636 \\
\hline 10.2364217 & -0.13636364 & 0.02727273 \\
\hline 10.3370607 & 0 & 0.00909091 \\
\hline 10.4664537 & 0.18181818 & 0.20454545 \\
\hline 10.5958466 & 0 & 0.13636364 \\
\hline 10.7827476 & -0.36363636 & -0.14545455 \\
\hline 10.9265176 & 0 & -0.24545455 \\
\hline 11.0271565 & 0.25 & 0.04545455 \\
\hline 11.2571885 & 0 & 0.00909091 \\
\hline 11.3865815 & -0.02272727 & 0 \\
\hline 11.4872204 & -0.02272727 & 0.10909091 \\
\hline 11.7028754 & -0.02272727 & 0.15909091 \\
\hline 11.8178914 & -0.02272727 & 0.20454545 \\
\hline 11.9329073 & -0.29545455 & 0.22727273 \\
\hline 12.1773163 & 0 & -0.24545455 \\
\hline 12.3354633 & 0.27272727 & 0.04545455 \\
\hline 12.4648562 & -0.2 & 0.09090909 \\
\hline 12.6230032 & -0.06818182 & -0.09090909 \\
\hline 12.9249201 & -0.15909091 & -0.27272727 \\
\hline 13.0255591 & 0 & -0.22727273 \\
\hline 13.2268371 & 0.33636364 & 0.18181818 \\
\hline
\end{tabular}

ISSN: 2523-2517

ECORFAN® Todos los derechos reservados

\begin{tabular}{|c|c|c|}
\hline 13.399361 & 0 & -0.13636364 \\
\hline 13.528754 & -0.31818182 & -0.04545455 \\
\hline 13.7444089 & -0.02272727 & -0.04545455 \\
\hline 13.9888179 & -0.34090909 & -0.27272727 \\
\hline 14.1182109 & 0 & -0.13636364 \\
\hline 14.3051118 & 0.5 & 0.27272727 \\
\hline 14.4057508 & 0 & 0.18181818 \\
\hline 14.5495208 & -0.5 & 0 \\
\hline 14.7364217 & 0 & 0 \\
\hline 14.8083067 & 0.18181818 & 0.02272727 \\
\hline 14.8658147 & 0 & -0.06363636 \\
\hline 15.0670927 & -0.5 & -0.18181818 \\
\hline 15.1533546 & 0 & 0 \\
\hline 15.2971246 & 0.61363636 & 0.20454545 \\
\hline 15.4552716 & 0 & 0.09090909 \\
\hline 15.5990415 & -0.61363636 & -0.09090909 \\
\hline 15.7140575 & 0 & -0.09090909 \\
\hline 15.8146965 & 0.34090909 & 0.05454545 \\
\hline 15.9584665 & 0 & -0.09090909 \\
\hline 16.0734824 & -0.56818182 & -0.24545455 \\
\hline 16.2028754 & 0 & -0.07272727 \\
\hline 16.3322684 & 0.68181818 & 0.20454545 \\
\hline 16.4760383 & 0 & 0.09090909 \\
\hline 16.6341853 & -0.75 & -0.27272727 \\
\hline 16.7635783 & 0 & -0.18181818 \\
\hline 16.8642173 & 0.54545455 & 0.15909091 \\
\hline 16.9936102 & 0 & -0.09090909 \\
\hline 17.1086262 & -0.59090909 & -0.27272727 \\
\hline 17.2236422 & 0 & -0.09090909 \\
\hline 17.3961661 & 0.77272727 & 0.27272727 \\
\hline 17.5111821 & 0 & 0.13636364 \\
\hline 17.6405751 & -0.75 & -0.19090909 \\
\hline 17.7699681 & 0 & -0.09090909 \\
\hline 17.884984 & 0.59090909 & 0.18181818 \\
\hline 18 & 0.04545455 & 0.16363636 \\
\hline
\end{tabular}

QUIÑONEZ-OSUNA, José Ramón, MARINONE-MOSCHETTO, Silvio Guido, FLORES-AGUILAR, María Dolores y CARMONADUARTE, Domingo. Evaluación de la energía mareomotriz en el norte del Golfo de California con fines de generación de energía eléctrica. Revista de Ingeniería Eléctrica. 2019. 